\title{
Effect of melatonin deficiency and disruption of its receptor signaling pathway on photosynthetic parameters and expression of chloroplast genes in plants of Arabidopsis thaliana under photooxidative stress
}

\author{
Bychkov I.A.*, Kudryakova N.V., Kusnetsov V.V. \\ K.A. Timiryazev Institute of Plant Physiology RAS, Moscow, Russia \\ *email:Ivan.a.b@mail.ru
}

Melatonin is a highly active biological substance with the well known antioxidant function in plants. However, its activity as a regulator of physiological processes is still of the great interest. The aim of this work was to study the regulatory role of melatonin under photooxidative stress in wild-type Arabidopsis, ecotype Columbia-0, and the mutants for melatonin synthesis (asmt) and signaling (cand2 and gpal). The two-weeksold plants were treated with $50 \mu \mathrm{M}$ of melatonin for three days and subjected to photostress $\left(600 \mu \mathrm{E} \mathrm{m}^{-2} \mathrm{~s}^{-1}\right)$ for 24 hours. Exposure of wild-type control plants to high light negatively affected the content of photosynthetic pigments, impaired the functioning of the reaction centers of photosystem II, and caused a decrease in the expression of photosynthetic genes and the content of endogenous melatonin. However, exogenous melatonin significantly reduced the damaging effect of light stress, as could be judged by physiological parameters, and maintained the expression of photosynthesis genes. A possible mechanism for maintaining the activity of chloroplast genes may be associated with the up regulation of the genes for chloroplast transcription apparatus since melatonin activated genes for plastid-encoded RNA polymerase and chloroplast nuclear-encoded RNA polymerase. The asmt mutant exhibited a reduced level of endogenous melatonin, much severe suppression of photosynthesis, and a greater sensitivity to exogenous melatonin. The cand2 and gpal mutants were insensitive to melatonin treatment: with the response to stress similar to that of Col-0, melatonin had no effect on physiological parameters, the expression of the some genes for transcription apparatus and photosynthetic genes. Thus, under conditions of photooxidative stress melatonin can act not only as an antioxidant, but also as a hormone-like substance, able to regulate the expression of chloroplast genes through the activity transcription apparatus. The data obtained confirms the role of CAND2 as a putative melatonin receptor. 